prévenus évidemment de la visite du contrôleur, ne pas pousser à fond la dernière traite précédant cette visite et laisser ainsi dans la mamelle un lait très riche en matière grasse qui, devant être récolté par le contrôleur à la première traite à laquelle celui-ci assiste, ne tend rien moins qu'à en élever le taux butyreux. C'est une question qui a beaucoup préoccupé les Américains du Nord et plusieurs travaux ont été faits sur le point de savoir dans quelle mesure une traite incomplète pouvait retentir sur le taux butyreux des traites qui suivent. A les lire, il semble qu'il en résulte un léger enrichissement en matière grasse de celles-ei.

L'opération de la traite incomplète est donc frauduleuse et e'est pourquoi certains règlements de Sociétés de contrôle exigent que le contrôleur assiste à la traite qui précèdera le début d'une épreuve, afin qu'il puisse s'assurer qu'elle a été poussée à fond.

Comme on peut en juger, la question de la périodicité du contrôle, fondamentale, a-t-il été dit, soulève un grand nombre de points intéressants. Les méthodes auxquelles le contrôle aura recours seront évidemment variables et chaque technique aura sa part d'approximation. Ce sont les circonstances qui en dicteront le choix. Pour engendrer un mouvement d'opinion en faveur du contrôle, on s'adressera d'abord à la plus simple, et à l'usage, rapidement les esprits, une fois convaincus de l'utilité de la méthode utilisée, seront les premiers à en apercevoir les points faibles et à en réclamer par suite l'amélioration.

Le contrôle laitier a le plus brillant avenir devant lui; ses buts sont parfaitement connus, les moyens d'y parvenir également. II reste à adapter ceux-ci aux circonstapces, tout en cherchant à obtenir, alors qu'on ne peut guère en éviter la simplification, qu'ils conduisent à des résultats toujours comparables entre eux et aussi près que possible de la vérité.

\title{
COMMENT DÉFINIR LA VALEUR EXACTE DES VACHES LAITIÈRES?
}

Par Vladimir SZCZEKIN-KROTOW

Directeur des Associations du Contrôle laitier près de la Société Agricole Centrale à Varsovie.

Introduction par M. le Prof. Dr Jean de ROSTAFINSKI.

L'auteur du présent article est bien connu en Pologne, parmi les éleveurs du bétail, comme directeur de l'Organisation des Associations du contrôle laitier. Il a done à sa disposition des données précises en chiffres et observations, et, en les étudiant, il enrichit la science en appliquant la méthode mathématique à la définition de phénomènes quotidiens qui n'ont aucunement éveillé l'attention jusqu'ici ou l'ont fait dans une mesure insuffisante. 
La question du rationnement des vaches laitières est un des plus graves problèmes physiologiques et économiques qui intéressent également le savant et l'éleveur. Mais, d'abord, il faut savoir combien de lait la vache donne annuellement. La définition du rendement annuel de la vache, calculé pendant l'année astronomique où l'année agricole ne convient pas pour comparer la valeur des vaches entre elles. Il suffit que la vache devienne stérile, ou qu'elle ne donne pas de lait pendant une certaine période de temps, ou qu'elle vêle tout de suite avant ou après le commencement de l'année d'observation, pour que le chiffre de sa lactation soit complètement différent. On sait aussi que les augmentations et les diminutions du rendement en relation avec la nourriture n'ont jamais été nettement définies.

C'est contre ces incertitudes que lutte l'auteur du présent article, en définissant dès le commencement le type de la vache normale et la corrélation existant entre la production annuelle (de vêlage à vêlage), et le plus haut rendement dans la même période de temps.

Je me permets donc de recommander ces remarques de M. SzczeкгN-Kвотоw au lecteur, car elles tentent de définir des questions très simples en apparence, mais très compliquées en réalité. Elles servent de base à nos études qui visent à l'augmentation du rendement des vaches et à l'appréciation exacte de celles-ci, ce qui est indispensable aux travaux de l'élevage, à une sélection éclairée et à une juste estimation des pédigrees des animaux domestiques. ***

Pour définir la valeur d'une vache au point de vue de son aptitude laitière, nous nous basons sur son rendement au cours de l'année agricole, ou au cours de l'ảnnée astronomique.

Mais en comparant les rendements annuels de quelques années successives, nous nous apercevons de grandes variations et nous restons à nous demander lequel de ces chiffres est exact; les courbes de la lactation nous fourniront la réponse demandée.

Il est clair que le rendement dépend de beaucoup de conditions : stérilité, âge, conditions du vêlage, nombre de vêlages, genre de nourriture, tendances individuelles, etc. Il en résulte que des changements apparaissent dans le rendement et que, sous leur influence, se modifie aussi la physionomie de la courbe de lactation qui change de sens et permet d'interpréter le rendement annuel.

Lorsque la lactation sera normale, le rendement annuel suffira à définir la valeur laitière de la vache ; cette courbe normale, ne dépendant que des tendances individuelles, nous sera fournie par les vaches qui, ayant vêlé, sont couvertes trois mois après le vêlage, sont bien nourries, et dont la période de lactation dure 300 jours. L'âge de la vache doit être aussi pris en considération.

Nous nous rendons compte que la production de la vache s'accroît 
au cours du premier mois après le vêlage, se maintient pendant quelque temps à un même niveau, puis subit sur son rendement quotidien une diminution de quelques litres au minimum, diminution progressive, depuis le jour de la monte jusqu'au jour d'interruption des traites.

La courbe de la lactation apparaîtrait donc comme suit :

Si nous définissons :

Le maximum de rendement journalier par $\mathrm{M}$,

Le rendement après le vêlage par $\mathrm{m}$,

Le rendement au moment de l'interruption des traites par $\mathbf{n}$,

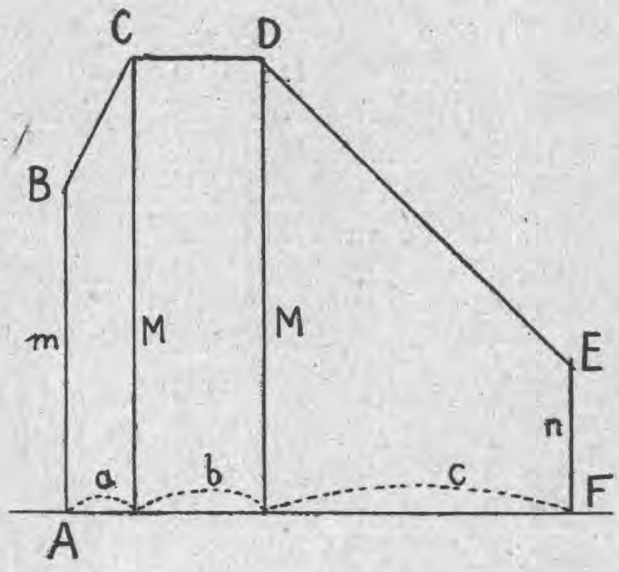

La période entre le vêlage et le rendement maximum par a,

La période du rendement maximum par $b$, et celle de la décroissance de production par e,

Le rendement annuel $\mathrm{P}$ répondra exactement à la surface du polygone A B C D E F, d'après la formule suivante :

$$
P=1 / 2 M(a+c)+M b+\frac{1}{2} m a+\frac{1}{2} n c
$$

ce qu'on peut écrire :

$$
P=1 / 2 M(a+b+c)+\frac{1}{2} M b+\frac{1}{2} m a+\frac{1}{2} n c
$$

Si nous considérons la somme $\mathrm{a}+\mathrm{b}+\mathrm{c}$ comme constante et égale à 300 (jours de traite), ainsi répartie: $\mathrm{a}=30$ jours, $\mathrm{b}=60$ jours, $\mathrm{c}=210$ jours, et si nous supposons $\mathrm{M}=16$ litres, $\mathrm{m}=12$ litres, $\mathrm{n}=4$ litres, le rendement annuel sera :

$$
2.400+480+180+420=3.480 \text { litres. }
$$

Il est facile de comprendre que les deux derniers termes du polynône forment un chiffre minime si nous les comparons au premier. Nous nous rendons compte d'ailleurs que le rendement maximum journalier est ce qui caractérise avant tout la période de la lactation. Nous sommes donc porté à croire qu'entre le rendement annuel et le maximum journalier il existe une corrélation exacte, et que cette corrélation suffira à caractériser le cours de la lactation car : 


$$
\frac{\mathrm{P}}{\mathrm{M}}=1 / 2(\mathrm{a}+\mathrm{b}+\mathrm{c})+1 / 2 \mathrm{~b}+1 / 2 \frac{\mathrm{m}}{\mathrm{M}} \mathrm{a}+1 / 2 \frac{\mathrm{n}}{\mathrm{M}} \mathrm{c},
$$

et approximativement $\frac{m}{M}=0,75$ et $\frac{n}{M}=0,25$. En cette conjecture $\frac{P}{M}$ devrait être égal à $150+30+11,25+26,25=217,5$.

Done la relation $\mathrm{P}$ : M représente la moitié de la somme du polynôme qui serait formé par la période de lactation + la période de lactation maximum, plus une partie (à peu près les trois quarts) de la période consécutive au vêlage où la lactation s'accroît + une partie (à peu près le quart) de la période de décroissance. Cette relation sera plus grande si la lactation et le rendement maximum durent plus longtemps, et lorsque les divergences entre $M, m$ et $n$ seront plus petites.

Ce premier cas est à observer chez les vaches bonnes laitières, le contraire se manifeste chez les vaches stériles, et, d'une manière moins accentuée, chez celles à rendement inférieur.

Le rendement est minime lorsque tout de suite après le vêlage la décroissance commence. En ce eas $\mathrm{P}$ (production annuelle) $=1 / 2 \mathrm{~m}$ $(\mathrm{a}+\mathrm{b}+\mathrm{c})$, et la relation de $\mathrm{P}$ à $\mathrm{M}$, que nous appellerons le «coefficient de la lactuosité ", sera de 150 . Au, contraire, ehez la vache pouvant tout de suite après le vêlage atteindre le maximum de rendement et le garder jusqu'à la fin de la période de la lactation, le eoefficient de la lactuosité serait de 300 . Donc,plus la relation de $\mathrm{P}$ à $\mathrm{M}$ approche 300 , mieux vaut la vache au point de vue laitier. En réalité ce coefficient n'atteint jamais le maximum, mème chez les vaches qui n'ont pas été couvertes au cours de leur lactation.

Ce coefficient décroît rapidement chez les vaches mal nourries; au contraire, si la nourriture est bonne, il s'élève.

Les comptes, établis à la base du "coefficient de lactuosité", seront déficients, si la vache a été mal nourrie après le vêlage et si la nourriture ne s'est améliorée que quelques mois après. En ce cas, le rendement maximum après le vêlage décroîtra rapidement, tandis que le coefficient se chiffrera par un nombre élevé. Le même cas se reproduira, si l'interruption de la traite a lieu trop tard.

Malgré ces inconvénients, l'usage du coefficient nous permet de nous rendre compte promptement de l'état d'upe étable et de sa valeur, ainsi que de vérifier les rendements annuels, et de constater les fautes qu'on a commises en les évaluant.

Nous avons mentionné déjà, dans la Gazeta Rolnicza (journal agricole) que les vaches jouissent annuellement de périodes d'affouragement abondant, et d'autres, au contraire, d'alimentation moins intense. Mieux nourries pendant l'hiver, elles le sont moins bien pendant l'été et l'automne. II s'en suit que les vaches qui ont vêlé au cours de l'hiver donnent proportionnellement plus de lait au 
commencement de la période de lactation et moins vers la fin de celle-ci, tandis que celles qui ont vêlé pendant l'été et l'automne donnent relativement plus de lait vers la fin de la période de lactation. Afin de le prouver, nous reproduisons ici les données de l'Association de contrôle laitier de Bimie?

\begin{tabular}{|c|c|c|c|c|c|c|c|c|c|c|c|c|}
\hline Mois du vellage & I & II & III & IV & v, & $\mathrm{vi}$ & vir & vilI & Ix & $\mathrm{x}$ & $\mathrm{xI}$ & XII \\
\hline Jours de traite & 314 & 330 & 312 & 295 & 289 & 288 & 299 & 293 & 292 & 295 & 287 & 86 \\
\hline $\begin{array}{l}\text { Production an- } \\
\text { nuelle . . }\end{array}$ & 3267 & 3130 & 3180 & 2920 & 2860 & 2730 & 2485 & 2480 & 2960 & 2660 & 3030 & \\
\hline $\begin{array}{c}\text { Miaximum jour- } \\
\text { nalier. }\end{array}$ & 16,8 & 14,2 & 15,5 & 15,8 & 16,2 & 16,4 & 15,3 & 13,5 & 16,5 & 13,5 & 10,9 & \\
\hline Rapport $\frac{P}{N}$. & 193 & 221 & 205 & 185 & 177 & 165 & 162 & 184 & 180 & 197 & 191 & \\
\hline
\end{tabular}

En étudiant les divers groupes d'animaux (ehaque groupe contient environ 20 sujets), nous pouvons nous apercevoir qu'aueun rendement annuel ne peut être considéré comme donnant l'indicatıon définitive de l'exacte capacité laitière des animaux. Les changements de fourrage, en influençant le nombre des jours de traite, la chute du maximum journalier, ont influencé aussi le rendement total annuel. Nous devons done nous en tenir à l'appréciation purement théorique du coefficient de lactuosité en le fixant à 217,5 comme indiqué plus haut.

De toutes les étables contrôlées pendant ces derniers temps, il n'y a que l'étable de Karoleys dont le ooefficient soit égal au coefficient théorique, car les vaches y ont été nourries d'une façon régulière et ont vêlé au cours de la même saison de l'année écoulée. Le coefficient de lactuosité y est de 216. Dans les autres étables, malgré un meilleur rendement, le coefficient n'atteint pas ce chiffre : à Rakovice il est de 204, à Seroczyn de 203, à Dabroszyn 200 ; dans les autres étables, il est moins élevé encore. Dans une étable, qui accusa le plus petit rendement de vaches qui ont vêlé et qui sont restées toute l'année à l'étable, il est de 145.

Jusque-là, nous avons parlé du rendement annuel comme si la période de lactation pouvait s'adapter rigoureusement à l'année du contrôle. En réalité, pour chiffrer le rendement annuel, nous sommes forcés de considérer la fin d'une période de lactation et le commencement de l'autre. En nous basant sur ce laps de temps, nous risquons fort de nous tromper; pour que le rendement annuel relevé caractérise entièrement la capacité de production de la vache, il faut que la vache se trouve dans des conditions normales pendant les deux lactations successives qui ont fourni les périodes servant à établir les caleuls. Au cas contraire, le chiffre relevé sera trop grand ou trop petit.

Des facteurs divers interviennent en effet, en particulier : $1^{\circ}$ stade 
de la lactation au début du contrôle, par conséquent époque de la saillie, et, par suite date du vêlage ; $2^{\circ}$ intervalle entre le vêlage et la saillie suivante.

Le chiffre fourni par le contrôle est maximum si le vêlage a lieu au commencement de l'année, et si la vache n'est pas couverte jusqu'à la fin de cette année. Le minimum sera au contraire enregistré, si les plus hauts chiffres de la lactation appartiennent à l'année qui a précédé le contrôle, donc si la vache vient à être couverte au commencement de l'année et vêle à la fin de celle-ci.

Les exemples suivants serviront à nous rendre compte de l'étendue de ces changements et de leur éloignement de la normale.

Pour évaluer le rendement normal, prenons l'exemple fourni au commencement du présent article, donc supposons $M=16, m=12$, $\mathrm{n}=4, \mathrm{a}=30, \mathrm{~b}=60, \mathrm{c}=210$. Rendement annuel : 3.480 litres.

Le maximum sera atteint quand il se rapprochera de $M$, et le rendement à 300 jours de traite deviendra 4.740 litres.

Le minimum apparaîtra lorsque $\mathrm{M}$ sera égal à $\mathrm{m}$, b égal à a qui se rapproche de 0 , de sorte que c será égal à 300 , et le rendement annuel sera de 2.400 litres.

Outre la montée tardive, un avortement ou un double vêlage peuvent occasionner un rendement anormal, et l'on comprend que ces divers facteurs pouvant exister sur l'une ou l'autre, ou sur les deux périodes des lactations suecessives entrant en compte pour le relevé du contrôle, donnent des combinaisons multiples susceptibles d'accroître ou de diminuer le rendement exact de l'animal. C'est, pratiquement, dans quelques cas seulement que le rendement annuel relevé coïncidera avec un rendement qu'on peut qualifier de normal.

Selon ce que seront les vêlages et les avortements de la vache au cours de quelques années successives, nous aurons des rendements annuels normaux ou anormaux. Mais il pourra arriver que le calcul le plus exact, donnant la moyenne relevée sur toutes les années de la vie de la vache, ne caractérisera pas son rendement normal; à plus forte raison, les comptes de quelques années seulement ne suffiront pas à le mettre en évidence.

Ainsi, puisque les avortements ou les montes tardives sont causés par de simples accidents, et ne sont pas la conséquence de défauts individuels, les comptes établis sur une base qui fait état de ces accidents ne peuvent caractériser la valeur laitière de la vache.

Nos conclusions seront donc les suivantes :

$10 \mathrm{Ni}$ la moyenne de quelques années, ni à plus forte raison le rendement total d'une année ne fournissent des données définitives pour évaluer la capacité laitière d'un sujet;

$2^{\circ}$ Pour évaluer le rendement de la vache, il est donc préférable de s'appuyer sur le maximum journalier ; 
$3^{\circ}$ En multipliant le maximum journalier par le coefficient de lactuosité qui, comme nous l'avons admis, est égal à 217,5 , nous pouvons évaluer eombien pourrait donner le sujet au cours d'une lactation normale;

$4^{\circ}$ Il est clair qu'en ce cas nous prenons comme base de calcul une moyenne qui ne pourra pas tenir compte de l'influence de l'individualité sur la lactation qui, d'ailleurs, à mon avis, n'est pas très importante;

$5^{\circ}$ Pour évaluer exactement la valeur d'une vache, on devra se baser sur la lactation normale au cours d'une période de temps représentée par le laps écoulé entre le jour du vêlage et le jour d'interruption de la traite - non pas pour l'année astronomique ou agricole - en prenant note de l'âge de la vache et de sa nourriture.

Pour finir, j'ajouterai que je ne considère nullement mes conclusions comme définitives, car je me rends nettement compte que quelques-unes d'entre elles sont hypothétiques et devront être vérifiées et étudiées ultérieurement.

\section{DE LA TuBerculose chez LA chèvRe(1)}

Par le vétérinaire en chef HONEKER, Freudenstadt (Wurtemberg).

Par suite du changement économique de l'Allemagne et surtout de l'insuffisance alimentaire, le lait de ehèvre est devenu un article très demandé. Aussi, la chèvre a pris de l'importance dans la pratique vétérinaire et a donné au praticien l'occasion d'examiner, beaucoup plus qu'auparavant, les maladies des caprins.

Une des plus intéressantes maladies infectieuses, tant au point de vue scientifique qu'au point de vue pratique, c'est la tuberculose de la chèvre, qui, à ma connaissance n'a jusqu'ici pas encore été étudiée à fond, et d'autant plus que sur son origine les opinions des spécialistes sont très divisées. Récemment, je reçus un travail d'un collègue berlinois, d'où il ressort qu'à propos de la tuberculose de la ehèvre les opinions divergent entre médecins et vétérinaires.

Il est dit que la chèvre est aussi sensible à la tuberculose humaine, que le bœuf. Etant placée dans d'aussi mauvaises conditions : entretien, fourrage, etc., elle prend la maladie tout aussi fortement que les bovins. Effectivement, ces derniers temps, plusieurs cas de tuberculose de la chèvre ont été reconnus.

Par contre, la chèvre réagit bien à l'infection tuberculeuse animale, mais à un moindre degré que le bœuf. 'L'augmentation de la tuberculose de la chèvre provient de l'énorme accroissement de l'élevage

(1) Rapport présenté au $2^{\circ}$ Congrès de l'élevage caprin (Fribourg, sept. 1925). 